\title{
Polynomial Stochastic Hybrid Systems
}

\author{
João Pedro Hespanha* \\ Center for Control Engineering and Computation \\ University of California, Santa Barbara, CA 93101
}

\begin{abstract}
This paper deals with polynomial stochastic hybrid systems (pSHSs), which generally correspond to stochastic hybrid systems with polynomial continuous vector fields, reset maps, and transition intensities. For pSHSs, the dynamics of the statistical moments of the continuous states evolve according to infinite-dimensional linear ordinary differential equations (ODEs). We show that these ODEs can be approximated by finite-dimensional nonlinear ODEs with arbitrary precision. Based on this result, we provide a procedure to build this type of approximations for certain classes of pSHSs. We apply this procedure for several examples of pSHSs and evaluate the accuracy of the results obtained through comparisons with Monte Carlo simulations. These examples include: the modeling of TCP congestion control both for long-lived and on-off flows; state-estimation for networked control systems; and the stochastic modeling of chemical reactions.
\end{abstract}

\section{Introduction}

Hybrid systems are characterized by a state-space that can be partitioned into a continuous domain (typically $\mathbb{R}^{n}$ ) and a discrete set (typically finite). For the stochastic hybrid systems considered here, both the continuous and the discrete components of the state are stochastic processes. The evolution of the continuous-state is determined by a stochastic differential equation and the evolution of the discrete-state by a transition or reset map. The discrete transitions are triggered by stochastic events much like transitions between states of a continuous-time Markov chains. However, the rate at which these transitions occur may depend on the continuous-state. The model used here for SHSs, whose formal definition can be found in Sec. 2] was introduced in [1] and is heavily inspired by the Piecewise-Deterministic Markov Processes (PDPs) in [2] Alternative models can be found in 345.

The extended generator of a stochastic hybrid system allows one to compute the time-derivative of a "test function" of the state of the SHS along solutions to the system, and can be viewed as a generalization of the Lie derivative for deterministic systems 12. Polynomial stochastic hybrid systems (pSHSs) are characterized by extended generators that map polynomial test functions into

\footnotetext{
* Supported by the National Science Foundation under grants CCR-0311084, ANI-
} 0322476 . 
polynomials. This happens, e.g., when the continuous vector fields, the reset maps, and the transition intensities are all polynomial functions of the continuous state. An important property of pSHSs is that if one creates an infinite vector containing the probabilities of all discrete modes, as well as all the multi-variable statistical moments of the continuous state, the dynamics of this vector are governed by an infinite-dimensional linear ordinary differential equation (ODE), which we call the infinite-dimensional moment dynamics (cf. Sec. 31).

SHSs can model large classes of systems but their formal analysis presents significant challenges. Although it is straightforward to write partial differential equations (PDEs) that express the evolution of the probability distribution function for their states, generally these PDEs do not admit analytical solutions. The infinite-dimensional moment dynamics provides an alternative characterization for the distribution of the state of a pSHS. Although generally statistical moments do not provide a description of a stochastic process as accurate as the probability distribution, results such as Tchebycheff, Markoff, or Bienaymé inequalities can be used to infer properties of the distribution from its moments.

In general, the infinite-dimensional linear ODEs that describe the moment dynamics for pSHSs are still not easy to solve analytically. However, sometimes they can be accurately approximated by a finite-dimensional nonlinear ODE, which we call the truncated moment dynamics. We show in Sec. 3 that, under suitable stability assumptions, it is in principle possible for a finite-dimensional nonlinear ODE to approximate the infinite-dimensional moment dynamics, up to an error that can be made arbitrarily small. Aside from its theoretical interest, this result motivates a procedure to actually construct these finite-dimensional approximations for certain classes of pSHSs. This procedure, which is described in Sec. 4 is applicable to pSHSs for which the (infinite) matrix that characterizes the moment dynamics exhibits a certain diagonal-band structure and appropriate decoupling between certain moments of distinct discrete modes. The details of this structure can be found in Lemma 1

To illustrate the applicability of the results, we consider several systems that appeared in the literature and that can be modeled by pSHSs. For each example, we construct in Sec. 5 truncated moment dynamics and evaluate how they compare with estimates for the moments obtained from a large number of Monte Carlo simulations. The examples considered include:

1. The modeling of network traffic under TCP congestion control. We consider two distinct cases: long-lived traffic corresponding to the transfer of files with infinite length; and on-off traffic consisting of file transfers with exponentially distributed lengths, alternated by times of inactivity (also exponentially distributed). These examples are motivated by [16].

2. The modeling of the state-estimation error in a networked control system that occasionally receives state measurements over a communication network. The rate at which the measurements are transmitted depends on the current estimation error. This type of scheme was shown to out-perform periodic transmission and can actually be used to approximate an optimal transmission scheme [78]. 
3. Gillespie's stochastic modeling for chemical reactions [9], which describes the evolution of the number of particles involved in a set of reactions. The reactions analyzed were taken from 1011] and are particularly difficult to simulate due to the existence of two very distinct time scales.

\section{Polynomial Stochastic Hybrid Systems}

A SHS is defined by a stochastic differential equation (SDE)

$$
\begin{aligned}
\dot{\mathbf{x}}=f(\mathbf{q}, \mathbf{x}, t)+g(\mathbf{q}, \mathbf{x}, t) \dot{\mathbf{n}}, \quad & f: \mathcal{Q} \times \mathbb{R}^{n} \times[0, \infty) \rightarrow \mathbb{R}^{n}, \\
& g: \mathcal{Q} \times \mathbb{R}^{n} \times[0, \infty) \rightarrow \mathbb{R}^{n \times k},
\end{aligned}
$$

a family of $m$ discrete transition/reset maps

$$
(\mathbf{q}, \mathbf{x})=\phi_{\ell}\left(\mathbf{q}^{-}, \mathbf{x}^{-}, t\right), \quad \phi_{\ell}: \mathcal{Q} \times \mathbb{R}^{n} \times[0, \infty) \rightarrow \mathcal{Q} \times \mathbb{R}^{n},
$$

$\forall \ell \in\{1, \ldots, m\}$, and a family of $m$ transition intensities

$$
\lambda_{\ell}(\mathbf{q}, \mathbf{x}, t), \quad \lambda_{\ell}: \mathcal{Q} \times \mathbb{R}^{n} \times[0, \infty) \rightarrow[0, \infty),
$$

$\forall \ell \in\{1, \ldots, m\}$, where $\mathbf{n}$ denotes a $k$-vector of independent Brownian motion processes and $\mathcal{Q}$ a (typically finite) discrete set. A SHS characterizes a jump process $\mathbf{q}:[0, \infty) \rightarrow \mathcal{Q}$ called the discrete state; a stochastic process $\mathbf{x}:[0, \infty) \rightarrow$ $\mathbb{R}^{n}$ with piecewise continuous sample paths called the continuous state; and $m$ stochastic counters $\mathbf{N}_{\ell}:[0, \infty) \rightarrow \mathbb{N}_{\geq 0}$ called the transition counters.

In essence, between transition counter increments the discrete state remains constant whereas the continuous state flows according to (11). At transition times, the continuous and discrete states are reset according to (2). Each transition counter $\mathbf{N}_{\ell}$ counts the number of times that the corresponding discrete transition/reset map $\phi_{\ell}$ is "activated." The frequency at which this occurs is determined by the transition intensities (3). In particular, the probability that the counter $\mathbf{N}_{\ell}$ will increment in an "elementary interval" $(t, t+d t]$, and therefore that the corresponding transition takes place, is given by $\lambda_{\ell}(\mathbf{q}(t), \mathbf{x}(t), t) d t$. In practice, one can think of the intensity of a transition as the instantaneous rate at which that transition occurs. The reader is referred to [1] for a mathematically precise characterization of this SHS. The following result can be used to compute expectations on the state of a SHS. For briefness, we omit a few technical assumptions that are straightforward to verify for the SHSs considered here:

Theorem 1 ([1] $)$. Given a function $\psi: \mathcal{Q} \times \mathbb{R}^{n} \times[0, \infty) \rightarrow \mathbb{R}$ that is twice continuously differentiable with respect to its second argument and once continuously differentiable with respect to the third one, we have that

$$
\frac{\mathrm{d} \mathrm{E}[\psi(\mathbf{q}(t), \mathbf{x}(t), t)]}{\mathrm{d} t}=\mathrm{E}[(L \psi)(\mathbf{q}(t), \mathbf{x}(t), t)],
$$




$$
\begin{aligned}
& \text { where } \forall(q, x, t) \in \mathcal{Q} \times \mathbb{R}^{n} \times[0, \infty) \\
& \qquad \begin{array}{r}
(L \psi)(q, x, t):=\frac{\partial \psi(q, x, t)}{\partial x} f(q, x, t)+\frac{\partial \psi(q, x, t)}{\partial t}+ \\
\quad+\frac{1}{2} \operatorname{trace}\left(\frac{\partial^{2} \psi(q, x)}{\partial x^{2}} g(q, x, t) g(q, x, t)^{\prime}\right)+ \\
\quad+\sum_{\ell=1}^{m}\left(\psi\left(\phi_{\ell}(q, x, t), t\right)-\psi(q, x, t)\right) \lambda_{\ell}(q, x, t),
\end{array}
\end{aligned}
$$

and $\frac{\partial \psi(q, x, t)}{\partial t}, \frac{\partial \psi(q, x, t)}{\partial x}$, and $\frac{\partial^{2} \psi(q, x)}{\partial x^{2}}$ denote the partial derivative of $\psi(q, x, t)$ with respect to $t$, the gradient of $\psi(q, x, t)$ with respect to $x$, and the Hessian matrix of $\psi$ with respect to $x$, respectively. The operator $\psi \mapsto L \psi$ defined by (5) is called the extended generator of the SHS.

We say that a SHS is polynomial ( $p S H S$ ) if its extended generator $L$ is closed on the set of finite-polynomials in $x$, i.e., $(L \psi)(q, x, t)$ is a finite-polynomial in $x$ for every finite-polynomial $\psi(q, x, t)$ in $x$. By a finite-polynomials in $x$ we mean a function $\psi(q, x, t)$ such that $x \mapsto \psi(q, x, t)$ is a (multi-variable) polynomial of finite degree for each fixed $q \in \mathcal{Q}, t \in[0, \infty)$. A pSHS is obtained, e.g., when the vector fields $f$ and $g$, the reset maps $\phi_{\ell}$, and the transition intensities $\lambda_{\ell}$ are all finite-polynomials in $x$.

\section{Examples of Polynomial Stochastic Hybrid Systems}

Example 1 (TCP long-lived [12]). The congestion window size $\mathbf{w} \in[0, \infty)$ of a long-lived TCP flow can be generated by a SHS with continuous dynamics $\dot{\mathbf{w}}=\frac{1}{R T T}$ and a reset map $\mathbf{w} \mapsto \frac{\mathbf{w}}{2}$, with intensity $\lambda(\mathbf{w}):=\frac{p \mathbf{w}}{R T T}$. The roundtrip-time $R T T$ and the drop-rate $p$ are parameters that we assume constant. This SHS has a single discrete mode that we omit for simplicity and its generator is given by

$$
(L \psi)(w)=\frac{1}{R T T} \frac{\partial \psi(w)}{\partial w}+\frac{p w(\psi(w / 2)-\psi(w))}{R T T},
$$

which is closed on the set of finite-polynomials in $w$.

Example 2 (TCP on-off [12]). The congestion window size $\mathbf{w} \in[0, \infty)$ for a stream of TCP flows separated by inactivity periods can be generated by a SHS with three discrete modes $\mathcal{Q}:=\{$ ss, ca, off $\}$, one corresponding to slowstart, another to congestion avoidance, and the final one to flow inactivity. Its continuous dynamics are defined by

$$
\dot{\mathbf{w}}= \begin{cases}\frac{(\log 2) \mathbf{w}}{R T T} & \mathbf{q}=\mathrm{ss} \\ \frac{1}{R T T} & \mathbf{q}=\mathrm{ca} \\ 0 & \mathbf{q}=\text { off }\end{cases}
$$

the reset maps associated with packet drops, end of flows, and start of flows are given by $\phi_{1}(\mathbf{q}, \mathbf{w}):=\left(\mathrm{ca}, \frac{\mathbf{w}}{2}\right), \phi_{2}(\mathbf{q}, \mathbf{w}):=(\mathrm{off}, 0)$, and $\phi_{3}(\mathbf{q}, \mathbf{w}):=\left(\mathrm{ss}, w_{0}\right)$, respectively; and the corresponding intensities are

$$
\lambda_{1}(\mathbf{q}, \mathbf{w}):=\left\{\begin{array}{ll}
\frac{p \mathbf{w}}{R T T} & \mathbf{q} \in\{\mathrm{ss}, \mathrm{ca}\} \\
0 & \mathbf{q}=\text { off }
\end{array} \quad \lambda_{2}(\mathbf{q}, \mathbf{w}):= \begin{cases}\frac{\mathbf{w}}{k R T T} & \mathbf{q} \in\{\mathrm{ss}, \mathrm{ca}\} \\
0 & \mathbf{q}=\text { off }\end{cases}\right.
$$




$$
\lambda_{3}(\mathbf{q}, \mathbf{w}):= \begin{cases}\frac{1}{\tau_{\text {off }}} & \mathbf{q}=\text { off } \\ 0 & \mathbf{q} \in\{\text { ss }, \mathrm{ca}\}\end{cases}
$$

The round-trip-time $R T T$, the drop-rate $p$, the average file size $k$ (exponentially distributed), the average off-time $\tau_{\text {off }}$ (also exponentially distributed), and the initial window size $w_{0}$ are parameters that we assume constant. The generator for this SHS is given by

$$
(L \psi)(q, w)= \begin{cases}\frac{(\log 2) w}{R T T} \frac{\partial \psi(\mathrm{ss}, w)}{\partial w}+\frac{p w(\psi(\mathrm{ca}, w / 2)-\psi(\mathrm{ss}, w))}{R T T}+\frac{w(\psi(\mathrm{off}, 0)-\psi(\mathrm{ss}, w))}{k R T T} & q=\mathrm{ss} \\ \frac{1}{R T T} \frac{\partial \psi(\mathrm{ca}, w)}{\partial w}+\frac{p w(\psi(\mathrm{ca}, w / 2)-\psi(\mathrm{ca}, w))}{R T T}+\frac{w(\psi(\mathrm{off}, 0)-\psi(\mathrm{ca}, w))}{k R T T} & q=\mathrm{ca} \\ \frac{\psi\left(\mathrm{ss}, w_{0}\right)-\psi(\mathrm{off}, w)}{\tau_{\mathrm{off}}} & q=\mathrm{off}\end{cases}
$$

which is closed on the set of finite-polynomials in $w$.

Example 3 (Networked control system [7]). Suppose that the state of a stochastic scalar linear system $\dot{\mathbf{x}}=a \mathbf{x}+b \dot{\mathbf{n}}$ is estimated based on state-measurements received through a network. For simplicity we assume that state measurements are noiseless and delay free. The corresponding state estimation error $\mathbf{e} \in \mathbb{R}$ can be generated by a SHS with continuous dynamics $\dot{\mathbf{e}}=a \mathbf{e}+b \dot{\mathbf{n}}$ and one reset map $\mathbf{e} \mapsto 0$ that is activated whenever a state measurement is received. It was conjectured in [7] and later shown in [8] that transmitting measurements at a rate that depends on the state-estimation error is optimal when one wants to minimize the variance of the estimation error, while penalizing the average rate at which messages are transmitted. This motivates considering the following reset intensity $\lambda(\mathbf{e}):=\mathbf{e}^{2 \rho}, \rho \in \mathbb{N}_{\geq 0}$. This SHS has a single discrete mode that we omitted for simplicity and its generator is given by

$$
(L \psi)(e):=a e \frac{\partial \psi(e)}{\partial e}+\frac{b^{2}}{2} \frac{\partial^{2} \psi(e)}{\partial e^{2}}+(\psi(0)-\psi(e)) e^{2 \rho},
$$

which is closed on the set of finite-polynomials in $e$.

Example 4 (Decaying-dimerizing reaction set [1011]). The number of particles $\mathbf{x}:=\left(\mathbf{x}_{1}, \mathbf{x}_{2}, \mathbf{x}_{3}\right)$ of three species involved in the following set of decayingdimerizing reactions

$$
S_{1} \stackrel{c_{1}}{\longrightarrow} 0, \quad 2 S_{1} \stackrel{c_{2}}{\longrightarrow} S_{2}, \quad S_{2} \stackrel{c_{3}}{\longrightarrow} 2 S_{1}, \quad S_{2} \stackrel{c_{4}}{\longrightarrow} S_{3}
$$

can be generated by a SHS with continuous dynamics $\dot{\mathbf{x}}=0$ and four reset maps

$$
\phi_{1}(\mathbf{x}):=\left[\begin{array}{c}
\mathbf{x}_{1}-1 \\
\mathbf{x}_{2} \\
\mathbf{x}_{3}
\end{array}\right] \quad \phi_{2}(\mathbf{x}):=\left[\begin{array}{c}
\mathbf{x}_{1}-2 \\
\mathbf{x}_{2}+1 \\
\mathbf{x}_{3}
\end{array}\right] \quad \phi_{3}(\mathbf{x}):=\left[\begin{array}{c}
\mathbf{x}_{1}+2 \\
\mathbf{x}_{2}-1 \\
\mathbf{x}_{3}
\end{array}\right] \quad \phi_{4}(\mathbf{x}):=\left[\begin{array}{c}
\mathbf{x}_{1} \\
\mathbf{x}_{2}-1 \\
\mathbf{x}_{3}+1
\end{array}\right]
$$

with intensities $\lambda_{1}(\mathbf{x}):=c_{1} \mathbf{x}_{1}, \lambda_{2}(\mathbf{x}):=\frac{c_{2}}{2} \mathbf{x}_{1}\left(\mathbf{x}_{1}-1\right), \lambda_{3}(\mathbf{x}):=c_{3} \mathbf{x}_{2}$, and $\lambda_{4}(\mathbf{x}):=c_{4} \mathbf{x}_{2}$, respectively. Since the numbers of particles take values in the discrete set of integers, we can regard the $\mathbf{x}_{i}$ as either part of the discrete or continuous state. We choose to regard them as continuous variables because we are interested in studying their statistical moments. In this case, the SHS has a single discrete mode that we omit for simplicity and its generator is given by

$$
\begin{gathered}
(L \psi)(x)=c_{1} x_{1}\left(\psi\left(x_{1}-1, x_{2}, x_{3}\right)-\psi(x)\right)+\frac{c_{2}}{2} x_{1}\left(x_{1}-1\right)\left(\psi\left(x_{1}-2, x_{2}+1, x_{3}\right)-\psi(x)\right) \\
+c_{3} x_{2}\left(\psi\left(x_{1}+2, x_{2}-1, x_{3}\right)-\psi(x)\right)+c_{4} x_{2}\left(\psi\left(x_{1}, x_{2}-1, x_{3}+1\right)-\psi(x)\right),
\end{gathered}
$$

which is closed on the set of finite-polynomials in $x$. 


\section{Moment Dynamics}

To fully characterize the dynamics of a SHS one would like to determine the evolution of the probability distribution for its state $(\mathbf{q}, \mathbf{x})$. In general, this is difficult so a more reasonable goal is to determine the evolution of (i) the probability of $\mathbf{q}(t)$ being on each mode and (ii) the moments of $\mathbf{x}(t)$ conditioned to $\mathbf{q}(t)$. In fact, often one can even get away with only determining a few loworder moments and then using results such as Tchebycheff, Markoff, or Bienaymé inequalities to infer properties of the overall distribution.

Given a discrete state $\bar{q} \in \mathcal{Q}$ and a vector of $n$ integers $m=\left(m_{1}, m_{2}, \ldots, m_{n}\right) \in$ $\mathbb{N}_{\geq 0}^{n}$, we define the test-function associated with $\bar{q}$ and $m$ to be

$$
\psi_{\bar{q}}^{(m)}(q, x):=\left\{\begin{array}{ll}
x^{(m)} & q=\bar{q} \\
0 & q \neq \bar{q},
\end{array} \quad \forall q \in \mathcal{Q}, x \in \mathbb{R}^{n}\right.
$$

and the (uncentered) moment associated with $\bar{q}$ and $m$ to be

$$
\mu_{\bar{q}}^{(m)}(t):=\mathrm{E}\left[\psi_{\bar{q}}^{(m)}(\mathbf{q}(t), \mathbf{x}(t))\right] \quad \forall t \geq 0 .
$$

Here and in the sequel, given a vector $x=\left(x_{1}, x_{2}, \ldots, x_{n}\right)$, we use $x^{(m)}$ to denote the monomial $x_{1}^{m_{1}} x_{2}^{m_{2}} \cdots x_{n}^{m_{n}}$.

PSHSs have the property that if one stacks all moments in an infinite vector $\mu_{\infty}$, its dynamics can be written as

$$
\dot{\mu}_{\infty}=A_{\infty}(t) \mu_{\infty} \quad \forall t \geq 0,
$$

for some appropriately defined infinite matrix $A_{\infty}(t)$. This is because $\forall \bar{q} \in$ $\mathcal{Q}, m=\left(m_{1}, \ldots, m_{n}\right) \in \mathbb{N}_{\geq 0}^{n}$, the expression $\left(L \psi_{\bar{q}}^{(m)}\right)(q, x, t)$ is a finite-polynomial in $x$ and therefore can be written as a finite linear combination of test-functions (possibly with time-varying coefficients). Taking expectations on this linear combination and using (4), (17), we conclude that $\dot{\mu}_{\bar{q}}^{(m)}$ can be written as linear combination of uncentered moments in $\mu_{\infty}$, leading to (8). In the sequel, we refer to (8) as the infinite-dimensional moment dynamics. Analyzing (and even simulating) (8) is generally difficult. However, as mentioned above one can often get away with just computing a few low-order moments. One would therefore like to determine a finite-dimensional system of ODEs that describes the evolution of a few low-order models, perhaps only approximately.

When the matrix $A_{\infty}$ is lower triangular (e.g., as in Example 3 with $\rho=0$ ), one can simply truncate the vector $\mu_{\infty}$ by dropping all but its first $k$ elements and obtain a finite-dimensional system that exactly describes the evolution of the moments. However, in general $A_{\infty}$ has nonzero elements above the main diagonal and therefore if one defines $\mu \in \mathbb{R}^{k}$ to contain the top $k$ elements of $\mu_{\infty}$, one obtains from (8) that

$$
\dot{\mu}=I_{k \times \infty} A_{\infty}(t) \mu_{\infty}=A(t) \mu+B(t) \bar{\mu}, \quad \bar{\mu}=C \mu_{\infty},
$$


where $I_{k \times \infty}$ denotes a matrix composed of the first $k$ rows of the infinite identity matrix, $\bar{\mu} \in \mathbb{R}^{r}$ contains all the moments that appear in the first $k$ elements of $A_{\infty}(t) \mu_{\infty}$ but that do not appear in $\mu$, and $C$ is the projection matrix that extracts $\bar{\mu}$ from $\mu_{\infty}$. Our goal is to approximate the infinite dimensional system (8) by a finite-dimensional nonlinear ODE of the form

$$
\dot{\nu}=A(t) \nu+B(t) \bar{\nu}(t), \quad \bar{\nu}=\varphi(\nu, t),
$$

where the map $\varphi: \mathbb{R}^{k} \times[0, \infty) \rightarrow \mathbb{R}^{r}$ should be chosen so as to keep $\nu(t)$ close to $\mu(t)$. We call (10) the truncated moment dynamics and $\varphi$ the truncation function. We need the following two stability assumptions to establish sufficient conditions for the approximation to be valid.

Assumption 1 (Boundedness). There exist sets $\Omega_{\mu}$ and $\Omega_{\nu}$ such that all solutions to (8) and (10) starting at some time $t_{0} \geq 0$ in $\Omega_{\mu}$ and $\Omega_{\nu}$, respectively, exist and are smooth on $\left[t_{0}, \infty\right)$ with all derivatives of their first $k$ elements uniformly bounded. The set $\Omega_{\nu}$ is assumed to be forward invariant.

Assumption 2 (Incremental Stability). There exists a function $11 \in \mathcal{K} \mathcal{L}$ such that, for every solution $\mu_{\infty}$ to (8) starting in $\Omega_{\mu}$ at some time $t_{0} \geq 0$, and every $t_{1} \geq t_{0}, \nu_{1} \in \Omega_{\nu}$ there exists some $\hat{\mu}_{\infty}\left(t_{1}\right) \in \Omega_{\mu}$ whose first $k$ elements match $\nu_{1}$ and

$$
\|\mu(t)-\hat{\mu}(t)\| \leq \beta\left(\left\|\mu\left(t_{1}\right)-\hat{\mu}\left(t_{1}\right)\right\|, t-t_{1}\right), \quad \forall t \geq t_{1},
$$

where $\mu(t)$ and $\hat{\mu}(t)$ denote the first $k$ elements of the solutions to (8) starting at $\mu_{\infty}\left(t_{1}\right)$ and $\hat{\mu}_{\infty}\left(t_{1}\right)$, respectively.

Remark 1. Assumption 2] was purposely formulated without requiring $\Omega_{\mu}$ to be a subset of a normed space to avoid having to choose a norm under which the (infinite) vectors of moments are bounded.

The result that follows establishes that the difference between solutions to (8) and (10) converges to an arbitrarily small ball, provided that a sufficiently large but finite number of derivatives of these signals match point-wise. To state this result, the following notation is needed: We define the matrices $C^{i}(t), i \in \mathbb{N}_{\geq 0}$ recursively by

$$
C^{0}(t)=C, \quad C^{i+1}(t)=C^{i}(t) A_{\infty}(t)+\dot{C}^{i}(t), \quad \forall t \geq 0, i \in \mathbb{N}_{\geq 0},
$$

and the family of functions $L^{i} \varphi: \mathbb{R}^{k} \times[0, \infty) \rightarrow \mathbb{R}^{r}, i \in \mathbb{N}_{\geq 0}$ recursively by

$$
\left(L^{0} \varphi\right)(\nu, t)=\varphi(\nu, t), \quad\left(L^{i+1} \varphi\right)(\nu, t)=\frac{\partial\left(L^{i} \varphi\right)(\nu, t)}{\partial \nu}(A(t) \nu+B(t) \varphi(\nu, t))+\frac{\partial\left(L^{i} \varphi\right)(\nu, t)}{\partial t},
$$

$\forall t \geq 0, \nu \in \mathbb{R}^{k}, i \in \mathbb{N}_{\geq 0}$. These definitions allow us to compute time derivatives of $\bar{\mu}(\tau)$ and $\bar{\nu}(\tau)$ along solutions to (8) and (10), respectively, because

$$
\frac{\mathrm{d}^{i} \bar{\mu}(t)}{\mathrm{d} t^{i}}=C^{i}(t) \mu_{\infty}(t), \quad \frac{\mathrm{d}^{i} \bar{\nu}(t)}{\mathrm{d} t^{i}}=\left(L^{i} \varphi\right)(\nu(t), t), \quad \forall t \geq 0, i \in \mathbb{N}_{\geq 0} .
$$

\footnotetext{
${ }^{1}$ A function $\beta:[0, \infty) \times[0, \infty) \rightarrow[0, \infty)$ is of class $\mathcal{K} \mathcal{L}$ if $\beta(0, t)=0, \forall t \geq 0 ; \beta(s, t)$ is continuous and strictly increasing on $s, \forall t \geq 0$; and $\lim _{t \rightarrow \infty} \beta(s, t)=0, \forall s \geq 0$.
} 
Theorem 2 ([13]). For every $\delta>0$, there exists an integer $N$ sufficiently large for which the following result holds: Assuming that for every $\tau \geq 0, \mu_{\infty} \in \Omega_{\mu}$

$$
C^{i}(\tau) \mu_{\infty}=\left(L^{i} \varphi\right)(\mu, \tau), \quad \forall i \in\{0,1, \ldots, N\},
$$

where $\mu$ denotes the first $k$ elements of $\mu_{\infty}$, then

$$
\|\mu(t)-\nu(t)\| \leq \beta\left(\left\|\mu\left(t_{0}\right)-\nu\left(t_{0}\right)\right\|, t-t_{0}\right)+\delta, \quad \forall t \geq t_{0} \geq 0,
$$

along all solutions to (8) and (10) with initial conditions $\mu_{\infty}\left(t_{0}\right) \in \Omega_{\mu}$ and $\nu\left(t_{0}\right) \in \Omega_{\nu}$, respectively, where $\mu(t)$ denotes the first $k$ elements of $\mu_{\infty}(t)$.

\section{Construction of Approximate Truncations}

Given a constant $\delta>0$ and sets $\Omega_{\mu}, \Omega_{\nu}$, it may be very difficult to determine the integer $N$ for which the approximation bound (14) holds. This is because, although the proof of Theorem 2 is constructive, the computation of $N$ requires explicit knowledge of the function $\beta \in \mathcal{K} \mathcal{L}$ in Assumption 2 and, at least for most of the examples considered here, this assumption is difficult to verify. Nevertheless, Theorem 2 is still useful because it provides the explicit conditions (13) that the truncation function $\varphi$ should satisfy for the solution to the truncated system to approximate the one of the original system. For the problems considered here we require (13) to hold for $N=1$, for which (13) simply becomes

$$
C \mu_{\infty}=\varphi(\mu, \tau), \quad C A_{\infty}(\tau) \mu_{\infty}=\frac{\partial \varphi(\mu, \tau)}{\partial \mu} I_{k \times \infty} A_{\infty}(\tau) \mu_{\infty}+\frac{\partial \varphi(\mu, \tau)}{\partial t},
$$

$\forall \mu_{\infty} \in \Omega_{\mu}, \tau \geq 0$. Lacking knowledge of $\beta$, we will not be able to explicitly compute for which values of $\delta$ (14) will hold, but we will show by simulation that the truncation obtained provides a very accurate approximation to the infinite-dimensional system (8), even for such a small choice of $N$. We restrict our attention to functions $\varphi$ and sets $\Omega_{\mu}$ for which it is simple to use (15D) to explicitly compute truncated systems.

Separable truncation functions: For all the examples considered, we consider functions $\varphi$ of the form

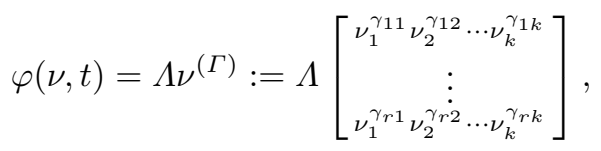

for appropriately chosen constant matrices $\Gamma:=\left[\gamma_{i j}\right] \in \mathbb{R}^{r \times k}$ and $\Lambda \in \mathbb{R}^{r \times r}$, with $\Lambda$ diagonal. In this case, (15) becomes

$$
\begin{aligned}
C \mu_{\infty} & =\Lambda \mu^{(\Gamma)} \\
C A_{\infty}(\tau) \mu_{\infty} & =\Lambda \operatorname{diag}\left[C \mu_{\infty}\right] \Gamma \operatorname{diag}\left[\mu_{1}^{-1}, \mu_{2}^{-1}, \ldots, \mu_{k}^{-1}\right] I_{k \times \infty} A_{\infty}(\tau) \mu_{\infty} .
\end{aligned}
$$


Deterministic distributions: A set $\Omega_{\mu}$ that is particularly tractable corresponds to deterministic distributions $\mathcal{F}_{\text {det }}:=\left\{P(\cdot ; q, x): x \in \Omega_{x}, q \in \mathcal{Q}\right\}$, where $P(\cdot ; q, x)$ denotes the distribution of $(\mathbf{q}, \mathbf{x})$ for which $\mathbf{q}=q$ and $\mathbf{x}=x$ with probability one; and $\Omega_{x}$ a subset of the continuous state space $\mathbb{R}^{n}$. For a particular distribution $P(\cdot ; q, x)$, the (uncentered) moment associated with $\bar{q}$ and $m \in \mathbb{N}_{\geq 0}^{n}$ is given by

$$
\mu_{\bar{q}}^{(m)}:=\int \psi_{\bar{q}}^{(m)}(\tilde{q}, \tilde{x}) P(d \tilde{q} d \tilde{x} ; q, x)=\psi_{\bar{q}}^{(m)}(q, x):= \begin{cases}x^{(m)} & q=\bar{q} \\ 0 & q \neq \bar{q},\end{cases}
$$

and therefore the vectors $\mu_{\infty}$ in $\Omega_{\mu}$ have this form. Although this family of distributions may seem very restrictive, it will provide us with truncations that are accurate even when the pSHSs evolve towards very "nondeterministic" distributions, i.e., with significant variance. For this set $\Omega_{\mu}$, (17) takes a particularly simple form and the following result provides a simple set of conditions to test if a truncation is possible.

Lemma $1([13])$. Let $\Omega_{\mu}$ be the set of deterministic distributions $\mathcal{F}_{\text {det }}$ with $\Omega_{x}$ containing some open ball in $\mathbb{R}^{n}$ and consider truncation functions $\varphi$ of the form (16). The following conditions are necessary for the existence of a function $\varphi$ of the form (16) that satisfies (15):

1. For every moment $\mu_{q_{\ell}}^{\left(m_{\ell}\right)}$ in $\bar{\mu}$ the polynomia $2 \sum_{\substack{i=1 \\ q_{i}=q_{\ell}}}^{\infty} a_{\ell, i} x^{\left(m_{i}\right)}$ must belong to the linear subspace generated by the polynomials

$$
\left\{\sum_{\substack{i=1 \\ q_{i}=q_{\ell}}}^{\infty} a_{j, i} x^{\left(m_{\ell}-m_{j}+m_{i}\right)}: 1 \leq j \leq k, q_{j}=q_{\ell}\right\} .
$$

2. For every moment $\mu_{q_{\ell}}^{\left(m_{\ell}\right)}$ in $\bar{\mu}$ and every moment $\mu_{q_{i}}^{\left(m_{i}\right)}$ in $\mu_{\infty}$ with $q_{i} \neq q_{\ell}$, we must have $a_{\ell, i}=0$. Here we are denoting by $a_{j, i}$ the $j$ th row, ith column entry of $A_{\infty}$.

Condition 1imposes a diagonal-band-like structure on the submatrices of $A_{\infty}$ consisting of the rows/columns that correspond to each moment that appears in $\bar{\mu}$. This condition holds for Examples 1, 2 and 3 but not for Example 4 However, we will see that the moment dynamics of this example can be simplified so as to satisfy this condition without introducing a significant error. Condition 2 imposes a form of decoupling between different modes in the equations for $\dot{\bar{\mu}}$. This condition holds trivially for all examples that have a single discrete mode. It does not hold for Example 2 but also here it is possible to simplify the moment dynamics to satisfy this condition without introducing a significant error.

\footnotetext{
${ }^{2}$ We are considering polynomials with integer (both positive and negative) powers.
} 

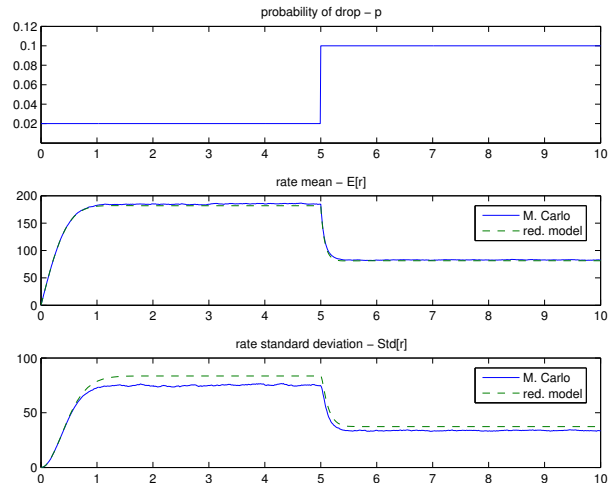

Fig. 1. Comparison between Monte Carlo simulations and the truncated model (18), (19) for Example 1 with $R T T=50 \mathrm{~ms}$ and a step in the drop-rate $p$ from $2 \%$ to $10 \%$.

\section{Examples of Truncations}

We now present truncated systems for the several examples considered before and discuss how the truncated models compare to estimates of the moments obtained from Monte Carlo simulations. All Monte Carlo simulations were carried out using the algorithm described in [1. Estimates of the moments were obtained by averaging a large number of Monte Carlo simulations. In most plots, we used a sufficiently large number of simulations so that the $99 \%$ confidence intervals for the mean cannot be distinguished from the point estimates at the resolution used for the plots. Ir is worth to emphasize that the results obtained through Monte Carlo simulations required computational efforts orders of magnitude higher than those obtained using the truncated systems.

Example 1 (TCP long-lived). Since for this system it is particularly meaningful to consider moments of the packet sending rate $\mathbf{r}:=\frac{\mathbf{w}}{R T T}$, we choose $\psi^{(m)}(w)=$ $\frac{w^{m}}{R T T^{m}}, \forall m \in \mathbb{N}_{\geq 0}$. We consider a truncation whose state contains the first and second moments of the sending rate. In this case, (9) can be written as follows:

$$
\left[\begin{array}{c}
\dot{\mu}^{(1)} \\
\dot{\mu}^{(2)}
\end{array}\right]=\left[\begin{array}{cc}
0 & -\frac{p}{2} \\
\frac{2}{R T T^{2}} & 0
\end{array}\right]\left[\begin{array}{c}
\mu^{(1)} \\
\mu^{(2)}
\end{array}\right]+\left[\begin{array}{c}
\frac{1}{R T T^{2}} \\
0
\end{array}\right]+\left[\begin{array}{c}
0 \\
-\frac{3 p}{4}
\end{array}\right] \bar{\mu}
$$

where $\bar{\mu}:=\mu^{(3)}$ evolves according to $\dot{\mu}^{(3)}=\frac{3 \mu^{(2)}}{R T T^{2}}-\frac{7 p \mu^{(4)}}{8}$. In this case, (17) has a unique solution $\varphi$, resulting in a truncated system given by (18) and

$$
\bar{\mu}=\varphi\left(\mu^{(1)}, \mu^{(2)}\right):=\frac{\left(\mu^{(2)}\right)^{\frac{5}{2}}}{\left(\mu^{(1)}\right)^{2}} .
$$

Figure 1 shows a comparison between Monte Carlo simulations and this truncated model. A step in the drop probability was introduced at time $t=5 \mathrm{sec}$ to show that the truncated model also captures well transient behavior. 
Example [ (TCP on-off). For this system we also consider moments of the sending rate $\mathbf{r}:=\frac{\mathbf{w}}{R T T}$ on the ss and ca modes, and therefore we use

$$
\begin{aligned}
\psi_{\mathrm{off}}^{(0)}(q, w) & = \begin{cases}1 & q=\mathrm{off} \\
0 & q \in\{\mathrm{ca}, \mathrm{ss}\}\end{cases} \\
\psi_{\mathrm{ca}}^{(m)}(q, w) & = \begin{cases}\frac{w^{m}}{R T^{m}} & q=\mathrm{ca} \\
0 & q \in\{\mathrm{ss}, \mathrm{off}\}\end{cases}
\end{aligned}
$$

We consider a truncation whose state contains the zeroth, first, and second moments of the sending rate. In this case, (9) can be written as follows:

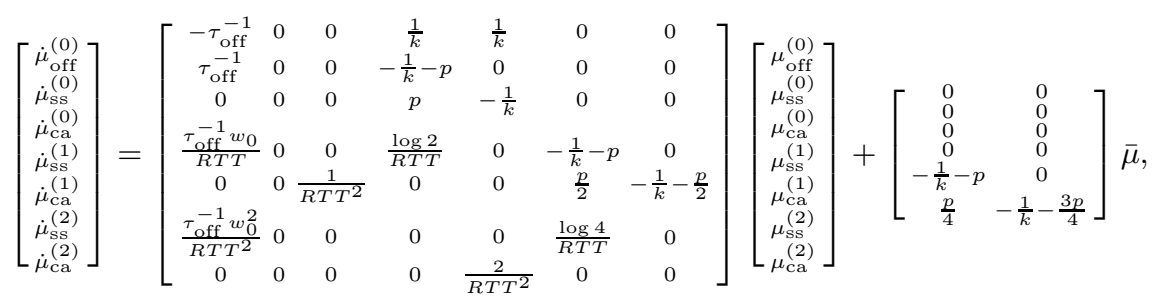

where $\bar{\mu}:=\left[\begin{array}{ll}\mu_{\mathrm{ss}}^{(3)} & \mu_{\mathrm{ca}}^{(3)}\end{array}\right]^{\prime}$ evolves according to

$$
\begin{aligned}
& \dot{\mu}_{\mathrm{ss}}^{(3)}=\frac{\tau_{\mathrm{off}}^{-1} w_{0}^{3}}{R T T^{3}} \mu_{\mathrm{off}}^{(0)}+\frac{\log 8}{R T T} \mu_{\mathrm{ss}}^{(3)}-\left(\frac{1}{k}+p\right) \mu_{\mathrm{ss}}^{(4)}, \\
& \dot{\mu}_{\mathrm{ca}}^{(3)}=\frac{3}{R T T^{2}} \mu_{\mathrm{ca}}^{(2)}+\frac{p}{8} \mu_{\mathrm{ss}}^{(4)}-\left(\frac{1}{k}+\frac{7 p}{8}\right) \mu_{\mathrm{ca}}^{(4)} .
\end{aligned}
$$

However, 21] does not satisfy condition 2 in Lemma 11 because the different discrete modes do not appear decoupled: $\dot{\mu}_{\mathrm{ss}}^{(3)}$ depends on $\mu_{\mathrm{off}}^{(0)}$, and $\dot{\mu}_{\mathrm{ca}}^{(3)}$ depends on $\mu_{\mathrm{ss}}^{(4)}$. For the purpose of determining $\varphi$, we ignore the cross coupling terms and approximate (21) by

$$
\dot{\mu}_{\mathrm{ss}}^{(3)} \approx \frac{\log 8}{R T T} \mu_{\mathrm{ss}}^{(3)}-\left(\frac{1}{k}+p\right) \mu_{\mathrm{ss}}^{(4)}, \quad \dot{\mu}_{\mathrm{ca}}^{(3)} \approx \frac{3}{R T T^{2}} \mu_{\mathrm{ca}}^{(2)}-\left(\frac{1}{k}+\frac{7 p}{8}\right) \mu_{\mathrm{ca}}^{(4)} .
$$

The validity of these approximations generally depends on the network parameters. When (22) is used, it is straightforward to verify that (17) has a unique solution $\varphi$, resulting in a truncated system given by (20) and

$$
\bar{\mu}=\varphi(\mu)=\left[\frac{\mu_{\mathrm{ss}}^{(0)}\left(\mu_{\mathrm{s}}^{(2)}\right)^{3}}{\left(\mu_{\mathrm{ss}}^{(1)}\right)^{3}} \frac{\left(\mu_{\mathrm{ca}}^{(0)}\right)^{\frac{1}{2}}\left(\mu_{\mathrm{ca}}^{(2)}\right)^{\frac{5}{2}}}{\left(\mu_{\mathrm{ca}}^{(1)}\right)^{2}}\right]^{\prime} .
$$

Figure 2 shows a comparison between Monte Carlo simulations and the truncated model (20), (23). The dynamics of the first and second order moments are accurately predicted by the truncated model. In preparing this paper, several simulation were executed for different network parameters and initial conditions. Figure 2 shows typical best-case (before $t=1$ ) and worst-case (after $t=1)$ results. 
Fig. 2. (left) Comparison between Monte Carlo simulations (solid lines) and the truncated model (20), (23) (dashed lines) for Example 2 with $R T T=50 \mathrm{~ms}, \tau_{\text {off }}=1 \mathrm{sec}$, $k=20.39$ packets (corresponding to $30.58 \mathrm{~KB}$ files broken into 1500 bytes packets, which is consistent with the file-size distribution of the UNIX file system reported in [14), and a step in the drop-rate $p$ from $10 \%$ to $2 \%$ at $t=1$ sec.
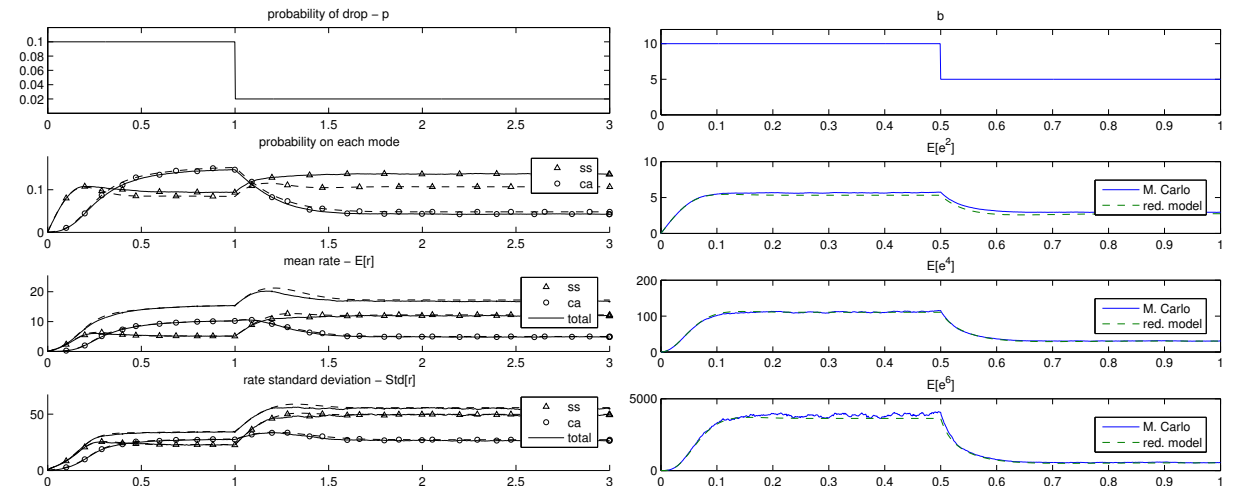

Fig. 3. (right) Comparison between Monte Carlo simulations (solid lines) and the truncated model (24), 25 (dashed lines) for Example 3 with $a=1, q=1$, and a step in the parameter $b$ from 10 to 2 at time $t=0.5 \mathrm{sec}$.

Example 3 (Networked control system). Now $\psi^{(m)}(e)=e^{m}, m \in \mathbb{N}_{\geq 0}$ and

$$
\left(L \psi^{(m)}\right)(e)=a m \psi^{(m)}(e)+\frac{m(m-1) b^{2}}{2} \psi^{(m-2)}(e)-\psi^{(m+2 \rho)}(e) .
$$

For $\rho=0$, the infinite-dimensional dynamics have a lower-triangular structure and therefore an exact truncation is possible. However, this case is less interesting because it corresponds to a reset-rate that does not depend on the continuous state and is therefore farther from the optimal [78]. We consider here $\rho=$ 1. In this case, the odd and even moments are decoupled and can be studied independently. It is straightforward to check that if the initial distribution of e is symmetric around zero, it will remain so for all times and therefore all odd moments are constant and equal to zero. Regarding the even moments, the smallest truncation for which condition 1 in Lemma 1 1 holds is a third order one, for which (9) can be written as follows:

$$
\left[\begin{array}{l}
\dot{\mu}^{(2)} \\
\dot{\mu}^{(4)} \\
\dot{\mu}^{(6)}
\end{array}\right]=\left[\begin{array}{ccc}
2 a & -1 & 0 \\
6 b^{2} & 4 a & -1 \\
0 & 15 b^{2} & 6 a
\end{array}\right]\left[\begin{array}{c}
\mu^{(2)} \\
\mu^{(4)} \\
\mu^{(6)}
\end{array}\right]+\left[\begin{array}{c}
b^{2} \\
0 \\
0
\end{array}\right]+\left[\begin{array}{c}
0 \\
0 \\
-1
\end{array}\right] \bar{\mu},
$$

where $\bar{\mu}:=\mu^{(8)}$ evolves according to $\dot{\mu}^{(8)}=-28 b^{2} \mu^{(6)}+8 a \mu^{(8)}-\mu^{(10)}$. It is straightforward to verify that (17) has a unique solution $\varphi$, resulting in a truncated system given by (24) and

$$
\bar{\mu}=\varphi\left(\mu^{(2)}, \mu^{(4)}, \mu^{(6)}\right)=\mu^{(2)}\left(\frac{\mu^{(6)}}{\mu^{(4)}}\right)^{3} .
$$


Figure 3 shows a comparison between Monte Carlo simulations and the truncated model (24), (25). The dynamics of the all the moments are accurately predicted by the truncated model. The nonlinearity of the underlying model is apparent by the fact that halving $b$ at time $t=0.5 \mathrm{sec}$, which corresponds to dividing the variance of the noise by 4 , only results in approximately dividing the variance of the estimation error by 2 .

Example 4 (Decaying-dimerizing reaction set). For this system the test functions are of the form $\psi^{\left(m_{1}, m_{2}, m_{2}\right)}(x)=x_{1}^{m_{1}} x_{2}^{m_{2}} x_{3}^{m_{3}}, \forall m_{1}, m_{2}, m_{3} \in \mathbb{N}_{\geq 0}$ and

$$
\begin{gathered}
\left(L \psi^{\left(m_{1}, m_{2}, m_{2}\right)}\right)(x)=c_{1} \sum_{i=0}^{m_{1}-1}\left(\begin{array}{c}
m_{1} \\
i
\end{array}\right)(-1)^{m_{1}-i} \psi^{\left(i+1, m_{2}, m_{3}\right)}(x) \\
+\frac{c_{2}}{2} \sum_{\substack{i, j=0 \\
(i, j) \neq\left(m_{1}, m_{2}\right)}}^{m_{1}, m_{2}}\left(\begin{array}{c}
m_{1} \\
i
\end{array}\right)\left(\begin{array}{c}
m_{2} \\
j
\end{array}\right)(-2)^{m_{1}-i}\left(\psi^{\left(i+2, j, m_{3}\right)}(x)-\psi^{\left(i+1, j, m_{3}\right)}(x)\right) \\
+c_{3} \sum_{\substack{i, j=0 \\
(i, j) \neq\left(m_{1}, m_{2}\right)}}^{m_{1}, m_{2}}\left(\begin{array}{c}
m_{1} \\
i
\end{array}\right)\left(\begin{array}{c}
m_{2} \\
j
\end{array}\right) 2^{m_{1}-i}(-1)^{m_{2}-j} \psi^{\left(i, j+1, m_{3}\right)}(x) \\
+c_{4} \sum_{\substack{i, j=0 \\
(i, j) \neq\left(m_{2}, m_{3}\right)}}^{m_{2}, m_{3}}\left(\begin{array}{c}
m_{2} \\
i
\end{array}\right)\left(\begin{array}{c}
m_{3} \\
j
\end{array}\right)(-1)^{m_{2}-i} \psi^{\left(m_{1}, i+1, j\right)}(x),
\end{gathered}
$$

where the summations result from the power expansions of the terms $\left(x_{i}-c\right)^{m_{i}}$. For this example we consider a truncation whose state contains all the first and second order moments for the number of particles of the first and second species. To keep the formulas small, we omit from the truncation the second moments of the third species, which does not appear as a reactant in any reaction and therefore its higher order statistics do not affect the first two. In this case, (9) can be written as follows:

$$
\left[\begin{array}{l}
\dot{\mu}^{(1,0,0)} \\
\dot{\mu}^{(0,1,0)} \\
\dot{\mu}^{(0,0,1)} \\
\dot{\mu}^{(2,0,0)} \\
\dot{\mu}^{(0,2,0)} \\
\dot{\mu}^{(1,1,0)}
\end{array}\right]=\left[\begin{array}{cccccc}
-c_{1}+c_{2} & 2 c_{3} & 0 & -c_{2} & 0 & 0 \\
-\frac{c_{2}}{2} & -c_{3}-c_{4} & 0 & \frac{c_{2}}{2} & 0 & 0 \\
0 & c_{4} & 0 & 0 \\
c_{1}-2 c_{2} & 4 c_{3} & 0 & 4 c_{2}-2 c_{1} & 0 & 0 \\
-\frac{c_{2}}{2} & c_{3}+c_{4} & 0 & \frac{c_{2}}{2} & -2 c_{3}-2 c_{4} & -c_{3} \\
c_{2} & -2 c_{3} & 0 & -\frac{3 c_{2}}{2} & 2 c_{3} & c_{2}-c_{1}-c_{3}-c_{4}
\end{array}\right]\left[\begin{array}{l}
\mu^{(1,0,0)} \\
\mu^{(0,1,0)} \\
\mu^{(0,0,1)} \\
\mu^{(2,0,0)} \\
\mu^{(0,2,0)} \\
\mu^{(1,1,0)}
\end{array}\right]+\left[\begin{array}{cc}
0 & 0 \\
0 & 0 \\
0 & 0 \\
-2 c_{2} & 0 \\
0 & c_{2} \\
\frac{c_{2}}{2} & -c_{2}
\end{array}\right] \bar{\mu},
$$

where $\bar{\mu}:=\left[\mu^{(3,0,0)} \mu^{(2,1,0)}\right]^{\prime}$ evolves according to

$$
\begin{aligned}
\dot{\mu}^{(3,0,0)}= & \left(-c_{1}+4 c_{2}\right) \mu^{(1,0,0)}+8 c_{3} \mu^{(0,1,0)}+\left(3 c_{1}-10 c_{2}\right) \mu^{(2,0,0)}+12 c_{3} \mu^{(1,1,0)} \\
& +\left(-3 c_{1}+9 c_{2}\right) \mu^{(3,0,0)}+6 c_{3} \mu^{(2,1,0)}-3 c_{2} \mu^{(4,0,0)} \\
\dot{\mu}^{(2,1,0)}= & -2 c_{2} \mu^{(1,0,0)}-4 c_{3} \mu^{(0,1,0)}+4 c_{2} \mu^{(2,0,0)}+4 c_{3} \mu^{(0,2,0)}+\left(c_{1}-2 c_{2}-4 c_{3}\right) \mu^{(1,1,0)} \\
- & \frac{5 c_{2} \mu^{(3,0,0)}}{2}+\left(4 c_{2}-2 c_{1}-c_{3}-c_{4}\right) \mu^{(2,1,0)}+4 c_{3} \mu^{(1,2,0)}+\frac{c_{2} \mu^{(4,0,0)}}{2}-2 c_{2} \mu^{(3,1,0)} .
\end{aligned}
$$


Table 1. Comparison between estimates obtained from Monte Carlo simulations and the truncated model for Example 4 The Monte Carlo data was taken from [1].

\begin{tabular}{lcccc}
\hline Source for the estimates & $\mathrm{E}\left[\mathbf{x}_{1}(0.2)\right]$ & $\mathrm{E}\left[\mathbf{x}_{2}(0.2)\right]$ & $\operatorname{StdDev}\left[\mathbf{x}_{1}(0.2)\right]$ & $\operatorname{StdDev}\left[\mathbf{x}_{2}(0.2)\right]$ \\
\hline 10,000 MC. simul. & 387.3 & 749.5 & 18.42 & 10.49 \\
model (27), (29) & 387.2 & 749.6 & 18.54 & 10.60 \\
\hline
\end{tabular}

This system does not satisfy condition 1 in Lemma@because the $\mu^{(1,0,0)}, \mu^{(0,1,0)}$ terms in the right-hand sides of (28) lead to monomials in $x_{1}$ and $x_{2}$ that do not exist in any of the polynomials $\left\{\sum_{i=1}^{\infty} a_{j, i} x^{\left(m_{\ell}-m_{j}+m_{i}\right)}: 1 \leq j \leq 6\right\}$. These terms can be traced back to the lowest-order terms in power expansions in (26) and disappear if we discard them. This leads to a simplified version of (27) for which condition 1 in Lemma 1 does hold, allowing us to find a unique solution $\varphi$ to (17), resulting in a truncated system given (27) and

$$
\bar{\mu}=\varphi(\mu)=\left[\left(\frac{\mu^{(2,0,0)}}{\mu^{(1,0,0)}}\right)^{3} \frac{\mu^{(2,0,0)}}{\mu^{(0,1,0)}}\left(\frac{\mu^{(1,1,0)}}{\mu^{(1,0,0)}}\right)^{2}\right]^{\prime} .
$$

Ignoring the lowest-order powers of $x_{1}$ and $x_{2}$ in the power expansions is valid when the populations of these species are high. In practice, the approximation still seems to yield good results even when the populations are fairly small. Figure 4 shows a comparison between Monte Carlo simulations and the truncated model (27), (29). The coefficients used were taken from [11, Example 1]: $c_{1}=1$, $c_{2}=10, c_{3}=1000, c_{4}=10^{-1}$. In Fig. 4(a) we used the same initial conditions as in [11 Example 1]: $\mathbf{x}_{1}(0)=400, \mathbf{x}_{2}(0)=798, \mathbf{x}_{3}(0)=0$. The match is very accurate, as can be confirmed from Table 1 The values of the parameters chosen result in a pSHS with two distinct time scales, which makes this pSHS computationally difficult to simulate ("stiff" in the terminology of [11]). Fig. 4(a) shows the evolution of the system on the "slow manifold," whereas Fig. 4(b) zooms in on the interval $\left[0,5 \times 10^{-4}\right]$ and shows the evolution of the system towards this manifold when it starts away from it at $\mathbf{x}_{1}(0)=800, \mathbf{x}_{2}(0)=100$, $\mathbf{x}_{3}(0)=200$. Figures $4(\mathrm{c}) 4(\mathrm{~d})$ shows another simulation of the same reactions but for much smaller initial populations: $\mathbf{x}_{1}(0)=10, \mathbf{x}_{2}(0)=10, \mathbf{x}_{3}(0)=5$. The truncated model still provides an extremely good approximation, with significant error only in the covariance between $\mathbf{x}_{1}$ and $\mathbf{x}_{2}$ when the averages and standard deviation of these variables get below one.

\section{Conclusions and Future Work}

In this paper, we showed that the infinite-dimensional linear moment dynamics of a pSHS can be approximated by a finite-dimensional nonlinear ODE with arbitrary precision. Moreover, we provided a procedure to build this type of approximation. The methodology was illustrated using a varied pool of examples, demonstrating its wide applicability. Several observations arise from these examples, which point to directions for future research: 


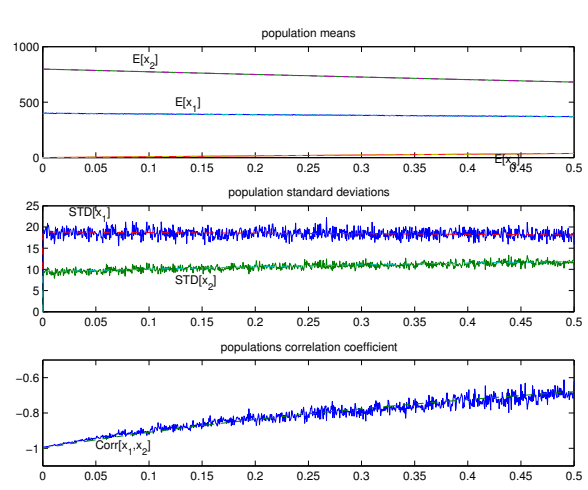

(a) Large population over a long time scale
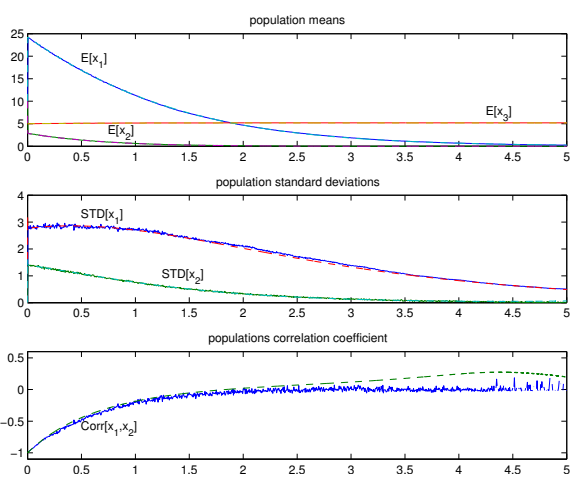

(c) Small population over a long time
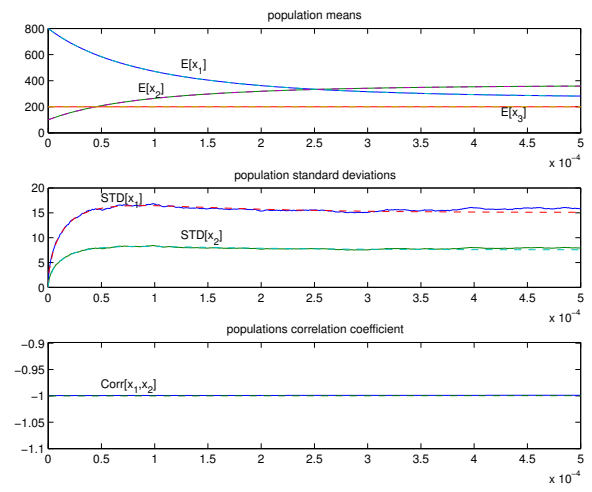

(b) Large population over a short time scale
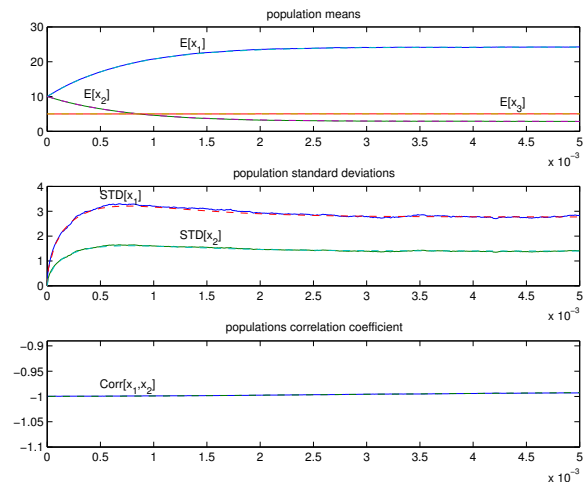

(d) Small population over short time scale

Fig. 4. Comparison between Monte Carlo simulations (solid lines) and the truncated model (27), 29] (dashed lines) for Example 4

1. In all the examples presented, we restricted our attention to truncation functions $\varphi$ of the form (16) and we only used deterministic distributions to compute $\varphi$. Mostly likely, better results could be obtained by considering more general distributions, which may require more general forms for $\varphi$.

2. The truncation of pSHSs that model chemical reactions proved especially accurate. This motivates the search for systematic procedures to automatically construct a truncated system from chemical equations such as (6). Another direction for future research consists of comparing the truncated models obtains here with those in [15]. 
An additional direction for future research consists of establishing computable bounds on the error between solutions to the infinite-dimensional moments dynamics and to its finite-dimensional truncations.

\section{References}

1. Hespanha, J.: Stochastic hybrid systems: Applications to communication networks. In Alur, R., Pappas, G., eds.: Hybrid Systems: Computation and Control. Number 2993 in Lect. Notes in Comput. Science. Springer-Verlag, Berlin (2004) 387-401

2. Davis, M.H.A.: Markov models and optimization. Monographs on statistics and applied probability. Chapman \& Hall, London, UK (1993)

3. Hu, J., Lygeros, J., Sastry, S.: Towards a theory of stochastic hybrid systems. In Lynch, N.A., Krogh, B.H., eds.: Hybrid Systems: Computation and Control. Volume 1790 of Lect. Notes in Comput. Science., Springer (2000) 160-173

4. Pola, G., Bujorianu, M., Lygeros, J., Benedetto, M.D.: Stochastic hybrid models: An overview. In: Proc. of IFAC Conf. on Anal. and Design of Hybrid Syst. (2003)

5. Bujorianu, M.: Extended stochastic hybrid systems and their reachability problem. In: Hybrid Systems: Computation and Control. Lect. Notes in Comput. Science. Springer-Verlag, Berlin (2004) 234-249

6. Bohacek, S., Hespanha, J., Lee, J., Obraczka, K.: A hybrid systems modeling framework for fast and accurate simulation of data communication networks. In: Proc. of ACM SIGMETRICS. (2003)

7. Xu, Y., Hespanha, J.: Communication logics for networked control systems. In: Proc. of 2004 Amer. Contr. Conf. (2004)

8. Xu, Y., Hespanha, J.: Optimal communication logics for networked control systems. In: Proc. of 43rd Conf. on Decision and Contr. (2004)

9. Gillespie, D.T.: A general method for numerically simulating the stochastic time evolution of coupled chemical reactions. J. Comp. Physics 22 (1976) 403-434

10. Gillespie, D., Petzold, L.: Improved leap-size selection for accelerated stochastic simulation. J. of Chemical Physics 119 (2003) 8229-8234

11. Rathinam, M., Petzold, L., Cao, Y., Gillespie, D.: Stiffness in stochastic chemically reacting systems: The implicit tau-leaping method. J. of Chemical Physics 119 (2003) 12784-12794

12. Hespanha, J.: A model for stochastic hybrid systems with application to communication networks. Submitted to the Int. Journal of Hybrid Systems (2004)

13. Hespanha, J.P.: Polynomial stochastic hybrid systems (extended version). Technical report, University of California, Santa Barbara, Santa Barbara (2004) Available

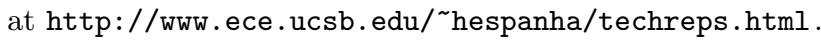

14. Irlam, G.: Unix file size survey - $1993 . \quad$ Available at http://www.base.com/gordoni/ufs93.html (1994)

15. Van Kampen, N.: Stochastic Processes in Physics and Chemistry. Elsevier (2001) 